\title{
Perfil dos farmacêuticos e farmácias em Santa Catarina: indicadores de estrutura e processo
}

\author{
José Benedito de França Filho', Cassyano Januário Correr*,2, Paula Rossignoli³, Ana Carolina \\ Melchiors' ${ }^{2}$, Fernando Fernández-Llimós ${ }^{4}$, Roberto Pontarolo ${ }^{2}$
}

${ }^{1}$ Conselho Regional de Farmácia de Santa Catarina, ${ }^{2}$ Grupo de Pesquisa em Prática Farmacêutica (GPPF), Universidade Federal do Paraná, ${ }^{3}$ Grupo de Pesquisa em Prática Farmacêutica (GPPF), Universidade Positivo, Farmácia Escola UnicenP, ${ }^{4}$ Faculdade de Farmácia, Universidade de Lisboa

*Correspondência:

C. J. Correr

Departamento de Farmácia

Universidade Federal do Paraná

Av. Lothario Meissner, 3400

80210-170 - Curitiba - PR, Brasil

E-mail: cassyano@ufpr.br
Este estudo teve por objetivo avaliar indicadores de estrutura e processo em farmácias comunitárias de Santa Catarina e as atitudes e percepções dos farmacêuticos referentes à atenção farmacêutica e satisfação profissional. Estudo transversal em 10\% das farmácias do estado, selecionadas aleatoriamente. Foram entrevistadas 258 farmácias. Os farmacêuticos estavam presentes em $88,4 \%$ delas, tinham média de idade de 31 anos $(D P=8,1)$ e eram em sua maioria mulheres (68\%). A média de farmacêuticos por farmácia foi de 1,4 $(D P=0,7)$ e de balconistas de 3,8 $(D P=4,5)$. Somente 11,4\% das farmácias dispunham de estrutura para atendimento privado de pacientes. Apesar de possuirem em média mais de duas fontes terciárias sobre medicamentos por farmácia, estas eram de baixa qualidade. As atividades relatadas pela maior parte dos farmacêuticos foram a dispensação (98,2\%), o registro de medicamentos controlados (90,8\%), aplicações de medicamentos injetáveis $(85,1 \%)$ e atendimentos de clientes no caixa (84,2\%). A maioria das farmácias de Santa Catarina não possui estrutura adequada à implantação de serviços de atenção farmacêutica. As barreiras identificadas para implementação destas mudanças não são diferentes das encontradas em outros países.
Uniterms

- Farmácia comunitária

- Farmácia/indicadores de qualidade

- Farmácia/estrutura de serviços

- Farmácia/processos

- Atenção farmacêutica

\section{INTRODUÇÃO}

A profissão farmacêutica encontra-se num período de profunda transformação, no Brasil e no mundo. Entidades como a Organização Mundial da Saúde (1993) e Conselho Federal de Farmácia (2001) vêm sistematicamente enfatizando a necessidade de maior integração do farmacêutico às equipes de saúde e provisão de serviços orientados ao paciente, num processo frequentemente referido como de re-profissionalização (Silva, Vieira, 2004). Neste novo contexto, o farmacêutico busca a "provisão responsável do tratamento farmacológico, com o propósito de 
alcançar resultados concretos que melhorem a qualidade de vida dos pacientes" (Hepler, Strand, 1990).

A atenção farmacêutica no Brasil começa a ser reconhecida após a elaboração do Consenso Brasileiro de Atenção Farmacêutica (Organização Panamericana da Saúde, 2002), que propõe um modelo de prática que contemple ações de promoção, proteção e recuperação da saúde. Entre os componentes da atenção farmacêutica refere-se a dispensação de medicamentos, o atendimento de distúrbios menores e o acompanhamento farmacoterapêutico como atividades que devem ser realizadas de forma sistemática e documentada pelo farmacêutico.

A dispensação é definida como o "ato profissional farmacêutico de proporcionar um ou mais medicamentos a um paciente, geralmente como resposta à apresentação de uma receita elaborada por um profissional autorizado. Nesse ato, o farmacêutico informa e orienta o paciente sobre o uso adequado do medicamento" (Brasil, 2001). O Acompanhamento Farmacoterapêutico constitui "um processo no qual o farmacêutico se responsabiliza pelas necessidades do usuário relacionadas ao medicamento, por meio da detecção, prevenção e resolução de Problemas Relacionados aos Medicamentos (PRM), de forma sistemática, contínua e documentada, com o objetivo de alcançar resultados definidos, buscando a melhoria da qualidade de vida do usuário" (Organização Panamericana da Saúde, 2002). Considerando esse novo cenário, as farmácias comunitárias, tanto de dispensação como manipulação, passam a necessitar de uma reestruturação e adequação para a provisão de serviços de atenção farmacêutica, além de padrões de qualidade para avaliação desses serviços.

As iniciativas para melhoria da qualidade das ações e serviços de saúde no Brasil vêm sendo desenvolvidas principalmente relacionados à organização do Sistema Único de Saúde, à área de análises clínicas e patológicas e aos hospitais privados. No âmbito das farmácias ainda são poucas as iniciativas de avaliação e ampliação da qualidade do setor farmacêutico. Como uma das várias formas de abordagem da melhoria da qualidade está a acreditação, com característica de atuar no sistema de organização, com enfoque na estrutura, nos processos e nos resultados (Silva, 2003).

Uma abordagem conceitual aceita mundialmente para o delineamento da avaliação dos serviços de saúde foi proposta por Donabedian (1966). Este autor organiza a avaliação dos serviços em estrutura, processo e resultado, sendo a estrutura correspondente aos recursos utilizados, incluindo recursos físicos, humanos, materiais, instrumentais normativos, administrativos, assim como as fontes de financiamento. O processo diz respeito às interações e procedimentos envolvendo profissionais de saúde e pacientes e os resultados (outcomes) são definidos como sendo a alteração no estado de saúde atribuível à intervenção em saúde. Os resultados receberam ainda de Kozma (1995) uma classificação, conhecida como modelo $E C H O$, subdividindo-os em econômicos, clínicos e humanísticos.

O objetivo deste estudo foi analisar indicadores de qualidade de estrutura e processo, e seus determinantes, em farmácias comunitárias de Santa Catarina e o perfil dos farmacêuticos presentes nestas farmácias em relação às atitudes e percepções referentes à atenção farmacêutica e satisfação profissional.

\section{MÉTODOS}

Estudo transversal exploratório realizado entre setembro e dezembro de 2004 envolvendo 10\% das farmácias comunitárias do estado de Santa Catarina. As farmácias foram selecionadas randomicamente a partir da listagem oficial de farmácias fornecida pelo Conselho Regional de Farmácia do estado. A listagem continha farmácias classificadas como de dispensação, manipulação e homeopáticas, totalizando 2.584 estabelecimentos.

As variáveis independentes consideradas referentes aos farmacêuticos foram sexo, idade, ensino superior público ou privado, cursos de pós-graduação realizados, vínculo com a farmácia, tempo de formado, conhecimento de língua estrangeira (inglês e espanhol) e conhecimento de informática. As variáveis independentes referentes às farmácias foram: tipo de farmácia (dispensação, manipulação ou homeopática), existência de área destinada à venda de artigos de conveniência (drugstore) e ser a farmácia de rede ou independente.

A escolha dos indicadores de estrutura baseou-se nas condições necessárias ao desenvolvimento da atenção farmacêutica, conforme descrito por diversos autores (Farris, Kirking, 1993; Machuca, Fernández-Llimós, Faus, 2003). Isso inclui disponibilidade de local de atendimento privado/ semiprivado, fontes de informação sobre medicamentos, recursos informáticos e de internet. O número de farmacêuticos e balconistas na equipe também foi pesquisado. Os processos analisados foram selecionados com base no documento sobre Boas Práticas de Farmácia do Conselho Federal de Farmácia (2002) e incluíram dispensação de medicamentos, verificação de pressão arterial, teste de glicemia, teste de colesterol/triglicerídeos, nebulização, administração de medicamentos injetáveis, manipulação alopática ou homeopática e acompanhamento farmacoterapêutico. Foram levantadas, ainda, atividades de treinamento de auxiliares, atendimento no caixa, controle de estoque e aquisição de medicamentos, atividades no setor financeiro da farmácia e registro de medicamentos controlados. 
A coleta desses dados foi realizada por meio de questionário estruturado auto-aplicável contendo 42 perguntas fechadas desenvolvido para a pesquisa. A maior parte das perguntas tinha como opção de resposta sim/ não. O conhecimento em língua estrangeira foi classificado em baixo/razoável/alto e em informática em baixo/ intermediário/avançado.

As informações dos farmacêuticos relativas à atenção farmacêutica foram coletadas em outro questionário contendo 13 perguntas agrupadas em 2 domínios: atitudes e percepções (10 perguntas) e satisfação (3 perguntas). Para atitudes e percepções foi utilizada como opção de respostas uma escala de freqüência tipo Likert de 1 a 5 (nunca, quase nunca, às vezes, quase sempre, sempre) e para satisfação uma escala de 1 a 5 (nada satisfeito, pouco satisfeito, satisfeito, bastante satisfeito, muito satisfeito). Para avaliação da confiabilidade (consistência interna) desta ferramenta calculou-se o alfa de Cronbach.

Os dados foram analisados com auxílio do software estatístico SPSS v.12.0 buscando-se associações entre variáveis dependentes e independentes. Foram considerados significativos valores de $\mathrm{p} \leq 0,05$.

\section{RESULTADOS}

Foram visitadas 258 farmácias. Destas, $61,6 \%$ eram farmácias independentes e 79,1\% não possuíam área de conveniência. A prática da venda de produtos não relacionados à saúde (conveniência) foi significativamente maior em farmácias de rede $(28,2 \%$ versus $16,3 \%, \mathrm{p}=0,022)$. Foram encontrados e entrevistados farmacêuticos em 228 das farmácias $(88,4 \%)$. A ausência do profissional foi mais freqüente em farmácias independentes $(17 \%)$ do que de rede $(3,1 \%)(p=0,02)$.

A média de idade dos farmacêuticos foi de 31 anos (IC 95\% 29,9-32,0) e 68\% (155) eram do sexo feminino. O tempo médio de formatura foi de 7,2 anos $(\mathrm{DP}=7,0)$ e eram graduados em instituições públicas $51,8 \%$ deles. A maioria (68\%) não havia cursado nenhuma pós-graduação. Não houve diferenças de idade $(\mathrm{p}=0,364)$ ou tempo de formatura $(p=0,433)$ entre aqueles que relataram ter cursado ou não qualquer pós-graduação. Com relação ao vínculo com a farmácia 57,1\% eram empregados e $42,9 \%$ sócios ou proprietários. Não houve diferença significativa de idade entre farmacêuticos empregados ou proprietários, no entanto, homens eram proprietários mais frequentemente do que mulheres $(54,1 \%$ versus $37,6 \%, \mathrm{p}=0,02)$. Com relação aos idiomas estrangeiros, $42,8 \%$ e $50,5 \%$ relataram domínio baixo de inglês e espanhol, respectivamente, e 47,7\% e 46,6\% domínio razoável. Com relação à informática, 87,2\% classificaram-se como usuários de nível intermediário ou avançado.

Questionados sobre a área de maior interesse profissional, $73,1 \%$ dos farmacêuticos afirmaram ser a área técnica, $15,9 \%$ a área administrativa e $11 \%$ ambas as áreas. Considerando os farmacêuticos que optaram por uma das áreas ( $n=202)$, não foram encontradas diferenças significativas considerando o vínculo com a farmácia, a instituição de formação, a idade e o tempo de formatura. Entre as mulheres, no entanto, foi mais freqüente o interesse pela área técnica $(86,4 \%$ versus $72,6 \%, \mathrm{p}=0,018)$.

A maior parte das farmácias $(90,4 \%)$ foi classificada como de dispensação, 5,3\% como manipulação, 3,1\% como dispensação e manipulação alopática e 1,3\% manipulação alopática e homeopática. Nenhuma farmácia visitada foi classificada exclusivamente como homeopática.

O número de farmacêuticos por farmácia foi de 1,4 (IC95\% 1,3-1,5) e de balconistas de 3,8 (IC95\% 3,2-4,4). Houve correlação positiva entre número de farmacêuticos e balconistas $(r=0,47, p<0,001)$. Os farmacêuticos relataram pagamento de comissão por vendas aos balconistas em $46,1 \%$ das farmácias.

Com relação às outras condições de estrutura, somente $11,4 \%$ (26) das farmácias possuíam área de atendimento privada/semiprivada para pacientes. Havia computador, impressora e acesso à internet disponíveis em 97,4\%, $89,9 \%$ e $78,1 \%$ das farmácias, respectivamente. A média de fontes terciárias sobre medicamentos por farmácia foi de 2,9 IC95\% 2,7-3,0. A maioria das farmácias (60,1\%) dispunha de 3 ou mais fontes e as farmácias de manipulação possuíam um número de fontes acima da média (4,7 IC95\% $3,8-5,5 ; p<0,05)$. Foi encontrada correlação negativa entre o tempo de formatura dos farmacêuticos e a quantidade de fontes disponíveis na farmácia $(\mathrm{r}=-0,174, \mathrm{p}<0,008)$ e a presença de fontes bibliográficas em inglês foi maior entre os farmacêuticos que relataram nível alto dessa língua, com relação aos que relataram nível baixo (19\% versus 5,3\%, $\mathrm{p}=0,03)$. As fontes terciárias mais citadas e a porcentagem de farmácias em que estão disponíveis encontram-se na Tabela I.

Com relação aos processos desenvolvidos pelos farmacêuticos nas farmácias (Tabela II), a dispensação de medicamentos representa o de maior freqüência $(98,2 \%)$, seguido pelo registro de medicamentos controlados (90,8\%), verificação de pressão arterial $(88,2 \%)$ e administração de medicamentos injetáveis $(85,1 \%)$. A realização de testes rápidos de glicemia e colesterol/triglicerídeos é pouco freqüente $(9,2 \%$ e $1,8 \%$, respectivamente), assim como a administração de medicamentos por nebulização (7,5\%).

A porcentagem de farmacêuticos que afirmaram realizar treinamento de auxiliares e acompanhamento farmacoterapêutico de pacientes também foi elevada 
TABELA I - Fontes terciárias disponíveis nas farmácias (n=228)

\begin{tabular}{lc}
\hline Fonte terciária & Farmácias que possuem n (\%) \\
\hline Dicionário de Especialidades Farmacêuticas - DEF & $223(97,8)$ \\
BPR - Guia de Remédios & $124(54,4)$ \\
Dicionário Terapêutico Guanabara & $118(51,8)$ \\
PR-Vademécum & $93(40,8)$ \\
Goodman \& Gilman. As bases Farmacológicas da Terapêutica & $43(18,9)$ \\
Martindale. The Extra Pharmacopea. & $12(5,3)$ \\
Drug Information for the Health Care Professional (USP-DI) & $10(4,4)$ \\
Outras & $29(12,7)$ \\
\hline
\end{tabular}

TABELA II - Atividades realizadas pelos farmacêuticos nas farmácias $(\mathrm{n}=228)$

\begin{tabular}{lc}
\hline Atividades & $\begin{array}{c}\text { Farmacêuticos } \\
\text { que as realizam }\end{array}$ \\
\hline Atividades de caráter técnico & $\mathrm{n}(\%)$ \\
Dispensação & $224(98,2)$ \\
Registro de medicamentos controlados & $207(90,8)$ \\
Verificação da Pressão Arterial & $201(88,2)$ \\
Teste de Glicemia & $21(9,2)$ \\
Teste de Colesterol/Triglicerídeos & $4(1,8)$ \\
Nebulização & $17(7,5)$ \\
Aplicação de injetáveis & $194(85,1)$ \\
Manipulação Magistral & $20(8,8)$ \\
Acompanhamento Farmacoterapêutico & $138(60,5)$ \\
Treinamento de auxiliares & $168(73,7)$ \\
Atividades de caráter administrativo & \\
Atendimento de clientes no caixa & $192(84,2)$ \\
Compra/Aquisição de medicamentos & $160(70,2)$ \\
Atividades do setor financeiro & $134(58,8)$ \\
\hline
\end{tabular}

(73,7\% e 60,5\%, respectivamente). Não foram observados aspectos relativos à sistematização e documentação dessas atividades ou ao método de acompanhamento farmacoterapêutico empregada.

Os farmacêuticos que relataram maior interesse pela área administrativa realizavam atividades do setor financeiro com maior freqüência em relação aqueles cujo interesse estava na área técnica $(77,8 \%$ versus $51,8 \%$, $\mathrm{p}=0,004)$. Entre os farmacêuticos sócios ou proprietários das farmácias, foi encontrada maior prevalência de atividades de compra/aquisição de medicamentos $(89,7 \%)$, setor financeiro $(91,8 \%)$ e treinamento de auxiliares $(86,6 \%)(p<0,001)$. Entre esses farmacêuticos foi também mais freqüente o relato de realização de acompa- nhamento farmacoterapêutico de pacientes $(69,1 \%$, $\mathrm{p}=0,02)$.

As atitudes e percepções na atenção farmacêutica foram analisadas em 220 farmacêuticos (Tabela III). Em uma escala de 1 (nunca) a 5 (sempre), a média das respostas foi de 3,7 ( $\mathrm{DP}=0,6)$. A análise da consistência interna do questionário revelou um alfa de Cronbach de 0,78 . Todos os itens mostraram correlação positiva significante $(\mathrm{r}>0,40, \mathrm{p}=0,01)$ com relação ao escore total do domínio. Não houve diferença significativa na média das respostas sobre atitudes e percepções em relação ao sexo, instituição de formatura, vínculo do farmacêutico com a farmácia e atuação em farmácia de rede. Foi encontrada diferença significativa, no entanto, entre farmacêuticos atuantes em farmácias com área de conveniência e sem área de conveniência $(3,5 \pm 0,8$ versus $3,7 \pm 0,5, \mathrm{p}=0,03)$. De acordo com o instrumento criado, quanto maior a pontuação, maior a atitude e percepção dos farmacêuticos.

As questões relativas à satisfação profissional, ligada à prática da atenção farmacêutica, foram preenchidas adequadamente por 227 farmacêuticos (Tabela IV). Em uma escala de 1 (nada satisfeito) a 5 (muito satisfeito), a média das respostas foi de 3,4 (DP=0,8). O alfa de Cronbach, que mede a confiabilidade do questionário, foi igual a 0,83 e a correlação (r) entre os itens e o escore total foi $\geq 0,85$ para todos os itens $(\mathrm{p}=0,01)$. O escore de satisfação dos farmacêuticos não foi significativamente diferente considerando a instituição de formação, o fato de a farmácia pertencer a uma rede ou possuir área de conveniência. Houve diferença significativa na satisfação entre homens e mulheres $(3,6$ $\pm 0,9$ versus $3,3 \pm 0,7, p=0,006$ ) e entre sócios/proprietários e empregados $(3,5 \pm 0,9$ versus $3,3 \pm 0,7, \mathrm{p}=0,018)$.

Os farmacêuticos que afirmaram realizar acompanhamento farmacoterapêutico de pacientes apresentaram escore significativamente maior com relação às atitudes $\mathrm{e}$ percepções $(3,5 \pm 0,8$ versus $3,1 \pm 0,7, \mathrm{p}<0,001)$ e satisfação profissional $(3,7 \pm 0,6$ versus $3,5 \pm 0,5, \mathrm{p}=0,017)$. 
TABELA III - Atitudes e percepções dos farmacêuticos com relação aos aspectos da atenção farmacêutica (n=220)*

\begin{tabular}{lcc}
\hline Com que freqüência você: & Média & DP \\
\hline $\begin{array}{l}\text { Sente-se responsável pelos bons ou maus resultados } \\
\text { do uso de medicamentos por seus pacientes? }\end{array}$ & 3,9 & 1,0 \\
Julga-se capaz de intervir no uso de medicamentos de um & & \\
paciente a partir da percepção de um problema? & 3,7 & 0,9 \\
$\begin{array}{l}\text { Percebe quando o paciente pode ter não entendido a } \\
\text { orientação dada por você? }\end{array}$ & 3,3 & 1,1 \\
$\begin{array}{l}\text { Percebe por parte do paciente confiança com relação } \\
\text { às orientações feitas por você? }\end{array}$ & 4,1 & 0,6 \\
$\begin{array}{l}\text { Sente-se trabalhando em equipe com o paciente? } \\
\text { Toma atitudes para resolver problemas referentes a }\end{array}$ & 3,7 & 0,8 \\
medicamentos, identificados em um paciente? & 3,9 & 1,0 \\
$\begin{array}{l}\text { Em casos como acima, procura saber como foi o resultado dessa ação? } \\
\text { Sente-se trabalhando em equipe com o médico? }\end{array}$ & 4,0 & 1,0 \\
$\begin{array}{l}\text { Sente-se seguro(a) para discutir com o médico algo sobre } \\
\text { o uso de medicamentos de um paciente? }\end{array}$ & 2,8 \\
$\begin{array}{l}\text { Sente-se seguro para enviar ao médico algo por escrito sobre } \\
\text { um paciente, datando e assinando o documento? }\end{array}$ & 3,6 & 1,1 \\
\hline
\end{tabular}

* Escala de respostas tipo Likert de 1 (nunca) a 5 (sempre). As perguntas foram formuladas de modo que quanto maior o escore da resposta, melhor a atitude e percepções do farmacêutico com relação à atenção farmacêutica. DP=desvio padrão.

TABELA IV - Satisfação dos farmacêuticos com relação a aspectos da atenção farmacêutica (n=227)*

\begin{tabular}{lcc}
\hline Como você classificaria atualmente sua satisfação com relação: & Média & DP \\
\hline A qualidade da sua relação com os pacientes que atende? & 3,5 & 0,8 \\
Ao papel desempenhado por você na equipe de profissionais & 3,4 & 0,9 \\
que atende seus clientes? & 3,3 & 1,0 \\
À sua carreira profissional de modo geral? & & \\
\hline
\end{tabular}

* Escala de respostas tipo Likert de 1 (nada satisfeito) a 5 (muito satisfeito). As perguntas foram formuladas de modo que quanto maior o escore da resposta, maior a satisfação. $\mathrm{DP}=$ desvio padrão

\section{DISCUSSÃO}

\section{Perfil das farmácias e dos farmacêuticos}

A maior parte das farmácias de Santa Catarina caracteriza-se como farmácia independente $(61,6 \%)$, sem área de conveniência $(79,1 \%)$ e cuja principal atividade é a dispensação de medicamentos industrializados $(90,4 \%)$. O comércio de produtos não relacionados à saúde (conveniência) foi mais freqüente em farmácias de rede do que em independentes, porém nestas últimas o profissional farmacêutico esteve mais ausente que nas primeiras.

Entre os farmacêuticos entrevistados a maioria (68\%) eram mulheres que demonstraram maior interesse pela área técnica que os homens $(86,4 \%$ vs. $72,6 \%$, $\mathrm{p}=0,018$ ). Esse resultado poderia ter relação com o fato dos farmacêuticos homens serem com mais freqüência proprietários de farmácia do que as mulheres.

A Atenção Farmacêutica é um modelo de prática profissional recente no cenário brasileiro e foi incluída como disciplina na maioria dos cursos de farmácia a partir de 2002, com a reforma curricular que instituiu a formação generalista do farmacêutico (Conselho Nacional de Educação, 2002). Considerando que o tempo médio de formatura dos farmacêuticos foi de 7,2 anos $(\mathrm{DP}=7,0) \mathrm{e}$ $68 \%$ não cursaram nenhuma pós-graduação (lato sensu ou strictu senso), espera-se que a maioria deles não possua formação específica em atenção farmacêutica. Outros autores já demonstraram falhas de formação nessa área (Silva, Vieira, 2004; Correr et al., 2004) e esses novos dados 
alertam para a necessidade de preenchimento dessa lacuna de formação.

A maioria dos farmacêuticos $(73,7 \%)$ entrevistados declarou realizar treinamento de auxiliares. Essa capacitação constitui uma estratégia de melhoria da qualidade da dispensação e promoção do uso racional de medicamentos (Naves et al., 2005). No entanto, experiências demonstraram que a formação nem sempre é suficiente na mudança de prática desses auxiliares (Oshiro, Castro, 2002). Avanços regulamentatórios na venda de medicamentos e reformulações das funções de farmacêuticos e auxiliares são também necessárias. Além disso, em 46,1\% das farmácias, os auxiliares são comissionados por venda de medicamentos o que poderia representar uma importante barreira para o desenvolvimento de atividades clínicas e boas práticas de farmácia.

\section{Indicadores de estrutura}

Os indicadores de estrutura pesquisados refletem as condições da farmácia para a realização de serviços de atenção farmacêutica. A verificação destes indicadores é necessária para avaliar se a estrutura existente é suficiente e que mudanças são necessárias para viabilizar a implantação do serviço (Silva, 2003). Para a prática da Atenção Farmacêutica e especialmente um dos seus componentes o acompanhamento farmacoterapêutico - é de fundamental importância a estrutura física com espaço para atendimento privado ou semiprivado. Este indicador de estrutura estava presente em apenas 11,4\% das farmácias, uma freqüência bem menor do que a relatada no estudo de Curitiba (92\%) (Correr et al., 2004) e bem maior do que 1\% relatado em estudo realizado na Espanha (Acosta-Gómez et al., 2003).

Outro indicador de estrutura para a prática da atenção farmacêutica é o acesso a fontes terciárias de informação sobre medicamentos, fundamental para a prestação de serviços de qualidade na dispensação ou acompanhamento farmacoterapêutico. Os livros disponíveis na maioria das farmácias pesquisadas (Dicionário de Especialidades Farmacêuticas, Guia de Remédios e Dicionário Terapêutico Guanabara) nem sempre atendem a demanda gerada por este tipo de serviço e, além disso, faz-se necessário a atualização anual destas fontes. Alguns autores (Loza et al., 1999; Barros, 2000) têm questionado consistentemente a qualidade da informação disponível nestas fontes, principalmente no que diz respeito a sua confiabilidade e completude. O nível de conhecimentos em informática (87,2\% se consideram intermediários ou avançados) juntamente com um aceitável conhecimento de línguas estrangeiras, ambos bastante maiores que os da Espanha (Andrés et al., 2000), por exemplo, poderiam favorecer o uso de fontes disponíveis na internet, comprovadamente úteis para a atenção farmacêutica (Silva-Castro et al., 2004).

\section{Indicadores de processo}

Com relação às atividades realizadas pelo farmacêutico na farmácia ainda é muito comum a divisão do trabalho entre atividades técnicas (ex. dispensação, acompanhamento farmacoterapêutico e verificação da pressão arterial) e administrativas (ex. aquisição de medicamentos). A dispensação de medicamentos $(98,2 \%)$ e o controle de compra e venda de medicamentos sujeitos a controle especial $(90,8 \%)$ são as atividades realizadas pelo maior número de farmacêuticos. Em um estudo realizado nos Estados Unidos com 2250 farmacêuticos em que se avaliou o tempo dedicado para determinadas atividades da farmácia, as atividades de dispensação foram as que ocuparam mais tempo $(56 \%)$ e as de acompanhamento farmacoterapêutico menos tempo (9\%) (Schommer et al., 2002).

Serviços como verificação de pressão arterial, teste de glicemia e colesterol/triglicerídeos são importantes para a verificação de resultados clínicos da farmacoterapia, um dos objetivos do acompanhamento farmacoterapêutico, além de possibilitarem ações de detecção precoce de casos suspeitos (rastreamento). Dentre estas atividades somente a verificação da pressão arterial é feita na maioria das farmácias $(88,2 \%)$. Cabe frisar que a realização desses procedimentos em farmácias não se encontra claramente regulamentada pela Agência Nacional de Vigilância Sanitária (1999)e não há legislação sanitária pertinente no Estado de Santa Catarina. A não regulamentação dessas atividades poderia atuar como um importante fator limitante à iniciativa por parte dos farmacêuticos de sua implantação nas farmácias.

Com relação a serviços de atenção farmacêutica, $60,5 \%$ dos farmacêuticos declararam que realizam acompanhamento farmacoterapêutico. Este, no entanto, exige condições de estrutura não disponíveis na maioria das farmácias. Além disso, informações sobre a sistematização e registro dessa atividade, que poderiam indicar se essa prática é efetivamente realizada, não foram levantadas, o que dificulta a avaliação desse resultado. Além disso, esses valores são bastante mais elevados que os encontrados em recentes estudos de outros países (Aguas et al., 2005).

\section{Atitudes e percepções}

De maneira geral, as questões que medem atitudes e percepções dos farmacêuticos com relação a aspectos da atenção farmacêutica obtiveram escores acima de 3 , que 
representa o ponto médio em uma escala de 1 (nunca) a 5 (sempre). A questão que obteve o mais baixo score $(2,8)$ foi a que trata da relação com o médico, demonstrando a dificuldade de relacionamento entre esses profissionais. Sabese, porém, que esta relação é essencial na instauração e manejo de uma farmacoterapia que beneficie o paciente (Pepe, de Castro, 2000). O escore mais alto e com menor dispersão foi atribuído à questão que trata da confiança do paciente com relação às orientações do farmacêutico $(4,1)$, seguido da questão que aborda a avaliação dos resultados de intervenções realizadas junto ao paciente $(4,0)$. O vínculo entre o paciente e o farmacêutico, bem como a busca por desfechos definidos, são pontos fundamentais para a prática do acompanhamento farmacoterapêutico, que só é passível de realização quando existe um trabalho em equipe com o paciente e cujo objetivo é a avaliação de resultados da farmacoterapia. Estas barreiras para a implementação de serviços cognitivos coincidem com trabalhos recentes de outros países (Roberts, 2003; Gastelurrutia et al., 2005).

Para pesquisas futuras sugere-se uma avaliação mais detalhada do processo de trabalho nestas farmácias, de que forma ocorre a integração da equipe e como são feitas a sistematização e o registro das atividades. Além disso, seria oportuna a realização desta mesma pesquisa em farmácias de outros estados, de modo a compor um perfil nacional das farmácias brasileiras e seus serviços.

\section{CONCLUSÃO}

Com os resultados desta pesquisa é possível concluir que a maioria das farmácias de Santa Catarina ainda não possui condições de estrutura adequadas para a efetiva implantação de serviços de atenção farmacêutica e principalmente do acompanhamento farmacoterapêutico. Além disso, a maioria dos farmacêuticos não possui formação específica em atenção farmacêutica, requisito essencial para a implantação desta nova prática.

As atividades realizadas pelos farmacêuticos encontram-se focadas demasiadamente nas tarefas administrativas. No entanto, as atitudes destes farmacêuticos com relação aos novos serviços centrados no paciente refletem pontuações maiores que as de sua atual satisfação. As barreiras identificadas para implementação destas mudanças não são diferentes das encontradas em outros países.

\section{ABSTRACT}

\section{Pharmacist's and pharmacies profiles in Santa Catarina: structure and process indicators}

quality indicators in community pharmacies in Santa Catarina (Brazil), and also the profile of pharmacists regarding their attitudes and perceptions of pharmaceutical care and professional satisfaction. A cross-sectional study was performed in 10\% of Santa Catarina pharmacies randomly chosen. 258 community pharmacies were surveyed. $88.5 \%$ pharmacists were in office in the time of the interview. In average, they were 31 years old $(S D=8.1)$ and mainly women $(68 \%)$. A ratio of $1.4(S D=0.7)$ pharmacists per pharmacy and $3.8(S D=4.5)$ assistants existed. Only $11.4 \%$ pharmacies had an adequate structure for private caring patients. Despite having an average of more than 2 tertiary drug information sources, most of them were low quality. Activities mentioned by the majority of pharmacists were dispensing (98.2\%), recording controlled drugs (90.8\%), injections administration (85.1\%), and helping patients on cash line (84.2\%). Most of pharmacies in Santa Catarina (Brazil) do not have the adequate structure for implementing pharmaceutical care services. Barriers identified to implement those services were not different to those communicated in other countries.

UNITERMS: Community pharmacy. Pharmacy/quality indicators. Pharmacy/services structure. Pharmacy/ process. Pharmaceutical care.

\section{AGRADECIMENTOS}

Os autores agradecem o apoio recebido do Conselho Regional de Farmácia de Santa Catarina para a realização deste estudo e aos seus fiscais que gentilmente colaboraram na coleta de dados.

\section{REFERÊNCIAS BIBLIOGRÁFICAS}

ACOSTA GÓMEZ, J.; ALZAGA LIZARRALDE, A.; ÁLVAREZ MOZOS, L.; GUDIEL URBANO, M.; FERNÁNDEZ-LLIMÓS, F. Estructura y servicios de atención farmacéutica ofrecidos en farmacias de Alcorcón (Madrid) y Bilbao. Seguim. Farmac., v.1, n.3, p.120-123, 2003.

AGÊNCIA NACIONAL DE VIGILÂNCIA SANITÁRIA. Resolução n³28, de 22 de julho de 1999. Dispõe sobre requisitos exigidos para dispensação de produtos de interesse à saúde em farmácias e drogarias. Brasília: Diário Oficial da União, 26 de julho de 1999. Disponível em: <http://e-legis.anvisa.gov.br/leisref/public/ showAct.php?id=1249>. Acesso em: 02 out. 2007. 
AGUAS, Y.; DE MIGUEL, E.; FERNANDEZ-LLIMÓS, F. El seguimiento farmacoterapéutico como innovación en las farmacias comunitarias de Badajoz (España). Seguim. Farmac., v.3, n.1, p.10-16, 2005.

ANDRÉS, N.F.; GARCÍA RIESTRA, R.; OCAMPO, M.T.; FERNÁNDEZ-LLIMÓS, F. Profile of the working group of members of the Pontevedra Society of Pharmacists. Pharm. Care Esp., v.2, p.20-27, 2000.

BARROS, J.A.C.A (Des)informação sobre medicamentos: o duplo padrão de conduta das empresas farmacêuticas. Cad. Saúde Pública, v.16, n.2, p.421-427, 2000.

BRASIL. Política Nacional de Medicamentos. Série C n.25. Brasília: Ministério da Saúde, 2001. 40p.

CONSELHO FEDERAL DE FARMACIA. Resolução, $n$. 357 de 20 de abril de 2001. Aprova o regulamento técnico das Boas Práticas de Farmácia. Brasília: Diário Oficial da União, 27 de Abril de 2001. Disponível em: <http://elegis.bvs.br/leisref/public/showAct.php?id=4123>. Acesso em: 02 out. 2007.

CONSELHO NACIONAL DE EDUCAÇÃO 2002. Resolução ${ }^{\circ}$ CNE/CES 2, de 19 de fevereiro de 2002. Brasília: Diário Oficial da União, 4 de março de 2001. Disponível em: <http://portal.mec.gov.br/cne/arquivos/ pdf/CES022002.pdf>.Acesso em: 02 out. 2007.

CORRER, C.J.; ROSSIGNOLI, P.; SOUZA, R.A.P.; PONTAROLO, R. Perfil de los farmacéuticos e indicadores de estructura y proceso en farmacias de Curitiba - Brasil. Seguim. Farmac., v.2, n.1, p.37-45, 2004.

DONABEDIAN, A. Evaluating the quality of medical care II. Milbank Memorial Fund., v.44, p.166-203, 1966.

FARRIS, K.B.; KIRKING, D.M. Assessing the quality of pharmaceutical care II. Application of concepts of quality assessment from medical care. Ann. Pharmac., v.27, p.215-223, 1993.

GASTELURRUTIA, M.A.; FERNANDEZ-LLIMOS, F.; GARCIA-DELGADO, P.; GASTELURRUTIA, P.; FAUS, M.J.; BENRIMOJ, S.I. Barriers and facilitators to the disseminaton and implementation of cognitive services in Spanish community pharmacies. Seguim Farmac, v.3, n.2, p.65-77, 2005.
HEPLER, C.D.; STRAND, L.M. Opportunities and responsibilities in the pharmaceutical care. Am. J. Hosp. Pharm., v.47, p.533-543, 1990.

KOZMA, C.M. Outcomes research and pharmacy practice. Am. Pharm., NS36, n.7, p.34-40, 1995.

LOZA, M.I.; CORDERO PUENTES, L.; FERNANDEZLLIMOS, F.; GARCIA CORRAL, P. Drug information sources used by community pharmacists in Galicia. Pharm. Care Esp., v.1, p.108-122, 1999.

MACHUCA, M.; FERNÁNDEZ-LLIMÓS, F.; FAUS, M.J. Método Dáder. Guia de seguimiento farmacoterapéutico. Granada: Editora Universidad de Granada, 2003. 47p.

NAVES, J.O.S.; MERCHAN-HAMANN, E.; SILVER, L.D. Orientação farmacêutica para DST: uma proposta de sistematização. Ciênc Saúde Coletiva, v.10, n.4, p.10051014, 2005.

ORGANIZAÇÃO MUNDIAL DA SAÚDE. El papel del farmacéutico en el sistema de atención de salud. Tókio, $1993.37 \mathrm{p}$.

ORGANIZAÇÃO PAN-AMERICANADA SAÚDE,(Org.). Consenso Brasileiro de Atenção Farmacêutica: Proposta. Brasília: OPAS, 2002. Disponível em: $<$ http:// www.opas.org.br/medicamentos $>$. Acesso em: 15 out. 2005.

OSHIRO, M.L.; CASTRO, L.L.C. Avaliação dos efeitos de uma intervenção educativa para promoção do uso da Terapia de Reidratação Oral (TRO) em trabalhadores de farmácias. Cad. Saúde Pública, v.18, n.1, p.287-297, 2002.

PEPE, V.L.E.; DE CASTRO, C.G.S.O.A interação entre prescritores, dispensadores e pacientes: informação compartilhada como possível benefício terapêutico. Cad. Saúde Pública, v.16, n.3, p.815-822, 2000.

ROBERTS, A.S.; HOPP, T.; SØRENSEN, E.W.; BENRIMOJ, S.I.; CHEN, T.F.; HERBORG, H.; WILLIAMS, K.; ASLANI, P. Understanding practice change in community pharmacy: a qualitative research instrument based on organisational theory. Pharm. World Sci., v.25, n.5, p.227-234, 2003. 
SCHOMMER, J.C.; PEDERSEN, C.A.; GAITHER, C.A.; DOUCETTE, W.R.; KRELING, D.H.; MOTT, D.A. Community Pharmacists' Work Activities in the United States During 2000. J. Am. Pharm. Assoc., v.42, p.399406, 2002.

SILVA, L.R.; VIEIRA, E.M. Conhecimento dos farmacêuticos sobre legislação sanitária e regulamentação da profissão. Rev. Saúde Pública, v.38, n.3, p.429-437, 2004.
SILVA, R.R. Acreditação de farmácias: a construção de um modelo. São Paulo, 2003. 197 p. [Tese de doutorado. Departamento de Práticas de Saúde Pública. Faculdade de Saúde Pública. Universidade de São Paulo].

SILVA-CASTRO, M.M.; BERMÚDEZ-TAMAYO, C.; GARCÍA GUTIÉRREZ, J.F.; JIMÉNEZ PERNETT, J.; TUNEU, L.; AZPILICUETA, I.; FERNÁNDEZLLIMÓS, F. Recursos web utilizados por los farmacéuticos para realizar atención farmacéutica. Seguim. Farmac., v.2, n.1, p.19-23, 2004.

Recebido para publicação em 18 de abril de 2007. Aceito para publicação em 24 de setembro de 2007. 